\title{
SCID-5-CV: CLINICAL VALIDITY OF THE DIFFERENTIAL DIAGNOSIS OF ANXIETY DISORDERS
}
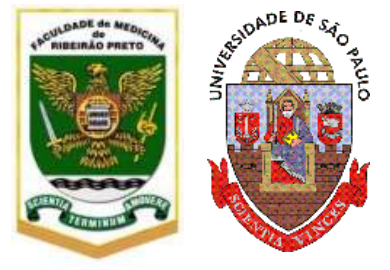

\section{INTRODUCTION}

- Clinical Validity refers to how well the diagnosis suggested by the diagnostic instruments is related to the presence or absence of a specific disease;

-The clinical accuracy of diagnostic instruments remains questionable, especially because it is rarely investigated, which is in part associated with the difficulty to establish a gold standard for comparison, a place that cannot be naturally fulfilled by clinical diagnosis;

- Unsatisfactory rates of clinical accuracy could be attributable to limitations of the instrument itself (such as the diagnostic algorithm and the restricted access to relevant information by the clinician), to diagnostic biases (connected with cultural issues of specific treatment units), and also to the training, expertise, and skill of the clinician in eliciting, understanding, and rating clinical material.

\section{- American Psychiatric Association (APA)}

\section{SCID-5-Clinician Version (SCID-5-CV)}

- short and reformatted version of SCID-5-RV

- for use by clinicians; can be used for research

- covers the most common diagnoses

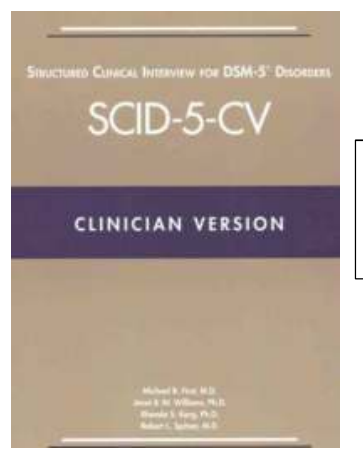

- Lack of psychometric studies to assess the clinical validity of SCID-5-CV

\section{OBJECTIVE}

- To assess the clinical validity of the DSM-5 Structured Clinical Interview (SCID-5-CV) for differencial diagosis of anxiety disorders in a sample of psychiatric in/outpatients with differents anxity disorders.

\section{METHODS}

\section{- Subjects}

$\Rightarrow 39$ psychiatric patients (inpatients/outpatients) with differents mood disorders

- $62 \%$ female; 36.97 years $(\mathrm{SD}=12.36)$

- Schooling of 15.29 years $(\mathrm{SD}=3.69)$

\section{- Evaluators}

$\Rightarrow 7$ psychiatrists and 5 psychologists

- formation time: 10 years $(\mathrm{SD}=4.3)$

- $100 \%$ experience in research and prior use of SCID

\section{- Collection and Analyses of Data}

$\Rightarrow$ evaluators conducted a SCID-5-CV (system of rotation). $\Rightarrow$ the clinical diagnosis was performed by the clinician responsible for the patient, through the LEAD procedure (longitudinal, evaluation performed by an expert, using all data available)

$\Rightarrow$ the SCID-5-CV sensitivity and specificity were calculated. Kappa $(\mathrm{K})$ coefficient was used, showing to be excellent when $>0.75$, satisfactory between 0.40 and 0.75 , and poor when $<0.40$

\section{RESULTS}

\begin{tabular}{lccc}
\hline \multicolumn{1}{c}{ Diagnosis } & \multicolumn{3}{c}{ SCID vs. Clinical diag. } \\
\cline { 2 - 4 } \multicolumn{1}{c}{ DSM5/ LEAD procedure } & Sensitivity & Specificity & Kappa \\
\hline Panic Disorder (current/past) & 0.88 & 0.96 & 0.73 \\
\hline Agoraphobia & 0.70 & 0.95 & 0.35 \\
\hline Social Anxiety Disorder & 0.85 & 0.96 & 0.71 \\
\hline General Anxiety Disorder & $\mathbf{0 . 7 2}$ & 0.94 & 0.61 \\
\hline Any Anxiety Disorder & $\mathbf{0 . 7 2}$ & $\mathbf{0 . 9 6}$ & $\mathbf{0 . 7 3}$ \\
\hline
\end{tabular}

Positive agreement for Any Anxiety Disorder: 73\%

Negative agreement for Any Anxiety Disorder: 96\%

\section{CONCLUSION}

- SCID-5-CV showed clinical validity to differentiate anxiety disorders, being an important resource to minimize false positive and negative rates in both clinical practice and research settings. 